\title{
Probing Microscopic Wetting Properties of Superhydrophobic Surfaces by Vibrated Micrometer-Sized Droplets
}

\author{
Alexandr Jonáš, ${ }^{*}$ Yasin Karadag, Nevin Tasaltin, Ibrahim Kucukkara, and Alper \\ Kiraz* \\ Department of Physics, Koç University, Rumelifeneri Yolu, 34450 Sariyer, Istanbul, Turkey \\ E-mail: ajonas@ku.edu.tr; akiraz@ku.edu.tr
}

\begin{abstract}
We determine contact angles of micrometer-sized $\mathrm{NaCl}$-water droplets on superhydrophobic surfaces by analyzing their lowest-order axisymmetric vibrational resonances driven by vertical oscillations of the surface. Fluorescence spectra of the dye-doped droplets excited by laser light feature whispering-gallery modes (WGMs) whose spectral widths depend on the droplet vibration amplitude, thus enabling precise measurements of the droplet mechanical resonant frequency. Following droplet size determination by WGM mode-matching, we calculate the contact angles from the dependence of the measured mechanical resonant frequency on the droplet size for two surfaces with different superhydrophobicity levels, and find a good correlation with the values measured by direct imaging of millimeter-sized droplets.
\end{abstract}


Superhydrophobic (SH) surfaces are gaining increasing attention because of their potential applications in liquid nanotechnology, e.g. in MEMS and optofluidic systems. ${ }^{1-4}$ The wetting properties of a SH surface can be described by the apparent contact angle $\theta$ that characterizes the overall energy balance of the liquid-solid-vapor interaction. ${ }^{5}$ Several techniques exist for measuring the value of $\theta$ over the macroscopic length scales of SH surfaces $(>100 \mu \mathrm{m}){ }^{6-8}$ however, these techniques do not allow probing of the variations of $\theta$ resulting from the micron-scale heterogeneity of the surface chemistry and/or topography. Alternative methods based on $\mathrm{AFM}^{9}$ or electron microscopy imaging ${ }^{10}$ are restricted by the accessible range of $\theta\left(\theta<90^{\circ}\right)$ or the ambient atmosphere humidity, respectively.

In this article, we introduce a novel method for the microscopic contact angle measurement that is based on the analysis of mechanical resonances of individual micrometer-sized liquid droplets supported by a vibrated SH surface. We exploit the spectroscopy of the optical whispering gallery modes (WGMs) excited by laser light in the fluorescently stained droplets to simultaneously identify the droplet mechanical resonances and sizes. The droplets act as optical microcavities ${ }^{4}$ and their fluorescence spectra feature WGMs whose spectral widths depend on the droplet vibration amplitude. By monitoring the WGM width as a function of the substrate oscillation frequency for droplets of different sizes, we identify their mechanical resonances at the frequencies of the maximal WGM broadening. Droplet size is then determined by mode-matching of the WGMs using the Lorenz-Mie scattering theory. For a given droplet, the precise value of the contact angle $\theta$ is subsequently obtained from independent measurements of the droplet mechanical resonance frequency and size using the previously developed theory for the axisymmetric sessile droplet oscillations. ${ }^{11,12}$ Using our technique we measure microscopic contact angles on two substrates with different levels of superhydrophobicity and find a good correlation with the average contact angles of millimetric droplets observed on the same surfaces.

We note that contact angle determination using droplet vibrational frequency measurement has been demonstrated previously only with millimeter-sized droplets. ${ }^{13}$ However, measurement of contact angles larger than $110^{\circ}$ was not reported in. ${ }^{13}$ Moreover, the experiments did not employ 
WGMs to simultaneously determine the mechanical resonance and size of a droplet with high precision.

(a)

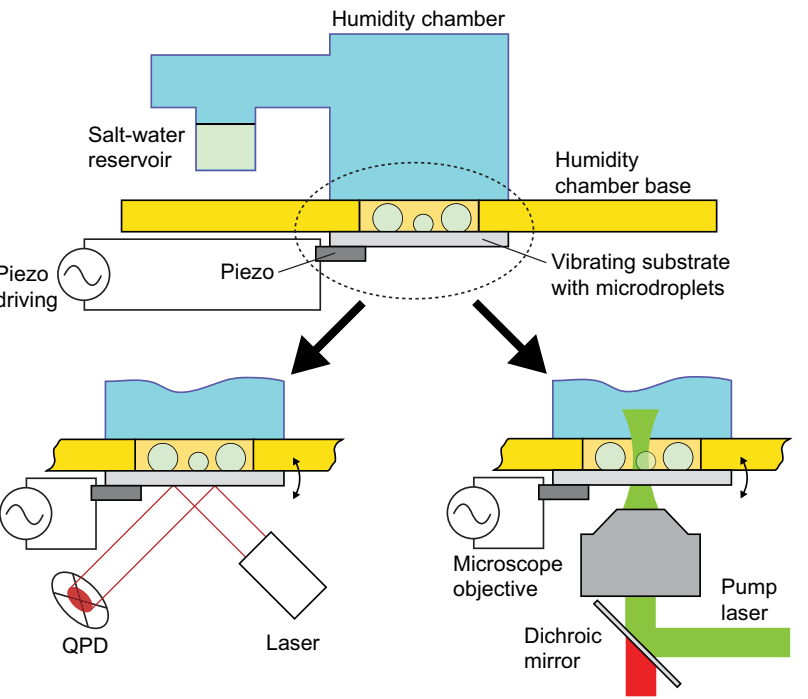

(b)

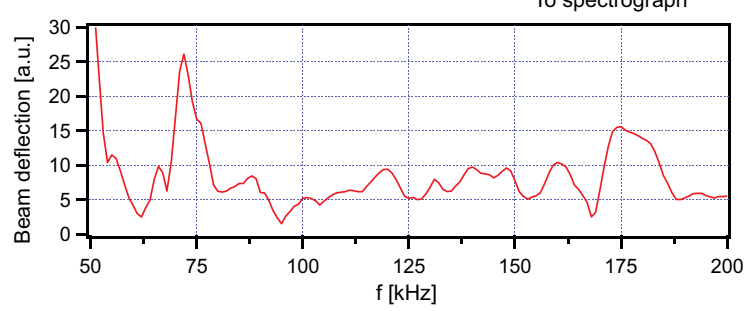

Figure 1: Experimental setup used for the microdroplet contact angle measurement. (a) Illustration of the controlled humidity sample chamber. [left] The frequency response of the mounted substrate to the harmonic PZT driving is characterized using an auxiliary collimated laser beam and a quadrant photodiode (QPD). [right] Actual detection of the microdroplet resonant frequency is carried out using inverted microscope configuration featuring a spectrograph. (b) Laser beam deflection from the substrate as a function of the driving frequency $f$. The beam deflection is directly proportional to the substrate vibration amplitude.

The model SH surfaces used in the experiments described here are prepared by spin-coating suspensions of hydrophobically-coated silica nanoparticles (Aeroxide LE1, average particle size: $14 \pm 3 \mathrm{~nm}$; Evonik) onto a cleaned cover glass as described previously. ${ }^{14}$ The superhydrophobicity level of the surface is adjusted by suspending the nanoparticles in different solvents (ethanol or chloroform at concentration of $50 \mathrm{mg} / \mathrm{ml}$ ) before spin-coating. Rhodamine B doped $\mathrm{NaCl}-$ water microdroplets (2.5 M NaCl, $100 \mu \mathrm{M}$ Rhodamine B) generated by an ultrasonic nebulizer are sprayed onto the SH surface in ambient atmospheric conditions. A piezoelectric transducer (PZT) 
that excites the droplet oscillations perpendicular to the plane of the cover glass is glued to the opposite surface of the cover glass using Entellan microscopy mounting medium (Merck Chemicals) so that it does not obstruct the observation of the droplets (see Figure 1a). Subsequently, the studied substrate bearing the microdroplets is attached to the bottom part of a home-made sample chamber whose relative water humidity is fixed at $84 \%$ with a saturated water solution of $\mathrm{KCl}$. Optical spectroscopic experiments are performed in the inverted microscope geometry (see Figure 1a, right). An air microscope objective (NA=0.8, 60x; Nikon) is used for tight focusing of the pump CW green laser ( $\lambda=532 \mathrm{~nm}, 4.5 \mathrm{~mW}$ max. power) onto the rim of a selected droplet. The fluorescence excited in the droplet is then collected by the same microscope objective and directed to a $500 \mathrm{~mm}$ monochromator with $1200 \mathrm{gr} / \mathrm{mm}$ grating (spectral resolution: $0.07 \mathrm{~nm}$, SpectraPro; Acton Research) that disperses the light before detection by a spectroscopic CCD camera (Pixis 100F; Princeton Instruments). A high voltage amplifier (WMA-300; Falco Systems) connected to the output of a function generator (33220A; Agilent) is used to drive the PZT with a $\sim 300 \mathrm{~V}$ peak-to-peak sinusoidal signal at varying frequencies.

For each prepared sample chamber, the vibrational response of the substrate was characterized by measuring the deflection of a laser beam reflected from the vibrating substrate with a quadrant photodiode (see Figure 1a, left). Figure 1b shows a typical vibrational response from a substrate used in the experiments. Considerable substrate vibration amplitudes are observed between 50$200 \mathrm{kHz}$. This is the critical frequency range that contains the lowest-order axisymmetric mechanical resonances of the micrometer-sized droplets studied here. ${ }^{11}$ Some variations were observed in the vibrational responses of different substrates used in the experiments, mainly due to variations in the elastic properties and the mounting of the cover glasses. However, the peaks featured in the substrate vibrational responses were always broad due to the amorphous nature of the cover glasses. Hence, approximately constant oscillatory forcing was ensured in the vicinity of the mechanical resonant frequencies of individual studied droplets.

Contact angle measurements relied on sweeping the piezo-driving frequency while recording the fluorescence spectra from individual microdroplets. Droplet mechanical resonances were then 
revealed after an analysis of the WGM widths as a function of the driving frequency. It should be noted that for each of the studied droplets, only a single mechanical resonant frequency was observed within the frequency range $50-200 \mathrm{kHz}$.
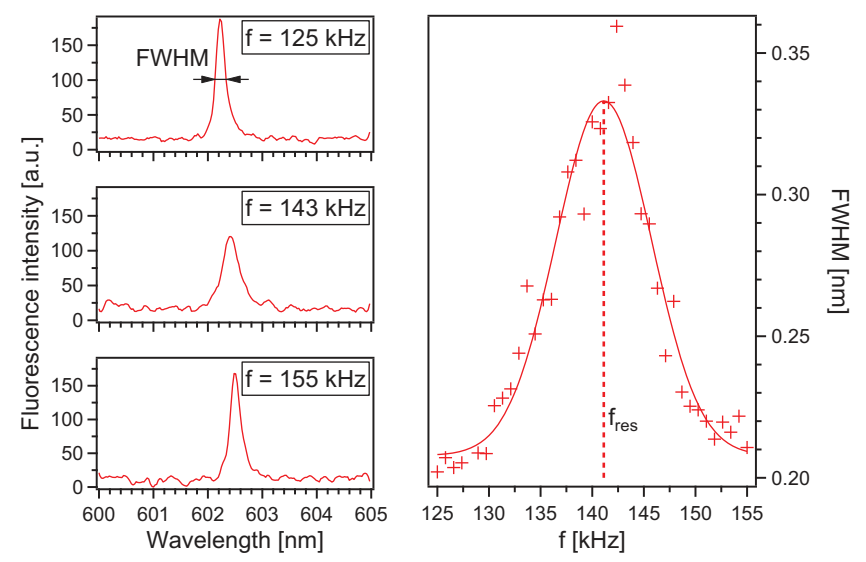

Figure 2: Response of surface-supported microdroplets to the harmonic oscillatory driving. (left) Fluorescence spectra of an oscillating droplet with spherical radius $R=5.0 \mu \mathrm{m}$ at three different driving frequencies $f$. The WGM centered at $602.3 \mathrm{~nm}$ is characterized by its full width at half maximum (FWHM). (right) FWHM as a function of $f$. The crosses indicate experimental data points, the continuous line is the Gaussian fit that gives the droplet resonant frequency $f_{\text {res }}^{\exp }=$ $141.1 \mathrm{kHz}$.

Figure 2 illustrates how the full width at half maximum (FWHM) of a WGM from an exemplary $\mathrm{NaCl}$-water microdroplet changes with the mechanical driving frequency $f$. The WGM in the fluorescence spectrum of the vibrated droplet displays a significant broadening when the mechanical resonant frequency is approached. This is caused by the intensified droplet shape oscillations at the resonance which lower the optical Q-factor of the droplet cavity. ${ }^{15}$ The actual resonance at $f_{\text {res }}^{\exp }=141.1 \mathrm{kHz}$ is clearly revealed by a Gaussian fit of the measured data. Vibration of sessile droplets has been recently extensively studied and various models predicting the droplet resonant frequencies have been proposed. ${ }^{16-18}$ For our case of vertically-driven micrometric spherical droplets whose shape is completely determined by surface tension with a negligible influence of gravity, the most appropriate model of oscillations is the one developed by Strani and 
Sabetta. ${ }^{11}$ Within this model, the lowest axisymmetric oscillation frequency $f_{\text {res }}^{\text {th }}$ is given by:

$$
f_{\mathrm{res}}^{\mathrm{th}}=(2 \pi)^{-1}\left[\frac{\gamma}{\rho R^{3} \lambda_{1}(\theta)}\right]^{1 / 2}
$$

where $\gamma, \rho$, and $R$ correspond to the surface tension of the liquid, the density of the liquid, and the droplet spherical radius, respectively. $\lambda_{1}(\theta)$ is the eigenvalue associated with the lowest-frequency mode (mode number 1 ) that strongly depends on the contact angle $\theta$. Consequently, for a droplet of known material properties and size, the value of $\theta$ can be determined using Eq. 1 and the measured resonant frequency $f_{\text {res }}^{\text {exp }}$.

Besides being extremely sensitive to small droplet vibrations, WGMs also allow an independent measurement of the droplet spherical radius $R$. Fluorescence spectra recorded in our experiments cover a spectral range of $39.1 \mathrm{~nm}$ containing at least three WGMs belonging to the same mode family identified by polarization and mode order. Within the framework of the Lorenz-Mie scattering theory, the value of $R$ can be obtained from direct mode-matching of these WGMs. ${ }^{15,19,20}$ In this approach, $R$ is determined by finding a set of droplet size parameters $a_{i}=(2 \pi R) / \lambda_{i}$ which provide the best match between the measured WGM spectral positions and those calculated for the droplet refractive index $n=1.3677$. Here, $\lambda_{i}$ denote the experimentally determined vacuum wavelengths of the WGMs contained within the given droplet fluorescence spectrum. For the case shown in Figure 2, mode-matching gives $R=5.0 \mu \mathrm{m}$. We estimate the uncertainty in $R$ values (mainly given by the uncertainty in the droplet refractive index) to be less than $100 \mathrm{~nm}$. Such precision cannot be obtained from the optical microscopy observation of the droplet as the identification of the droplet equatorial plane where its spherical radius can be measured is to large extent arbitrary.

Figure 3 shows the distributions of $f_{\text {res }}^{\exp }$ for microdroplets of various spherical radii $R$ supported by two different SH surfaces (LE1/ethanol coating - red circles, LE1/chloroform coating blue triangles). For both of the studied surfaces, the experimental data was recorded on several substrates prepared using the procedure described above and no significant substrate-to-substrate 


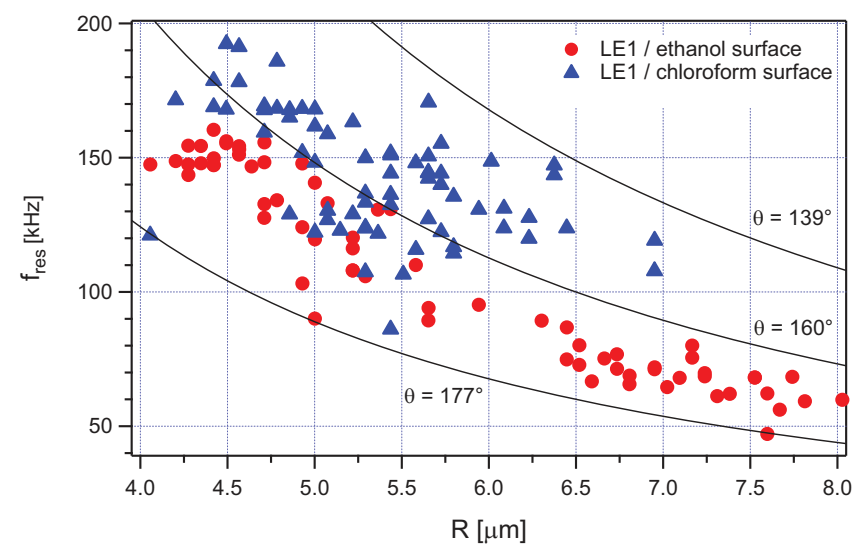

Figure 3: Dependence of the lowest-mode resonant frequency $f_{\text {res }}$ on the droplet size. The circles and triangles indicate experimental data points obtained for droplets supported by the LE1/ethanol and LE1/chloroform SH surfaces, respectively. The lines show the resonant frequency values calculated from Eq. 1 for the contact angles of $177^{\circ}$ (lower frequency limit), $160^{\circ}$ (frequency mid-range), and $139^{\circ}$ (upper frequency limit). Other calculation parameters for the NaCl-water microdroplet: surface tension $\gamma=74.62 \mathrm{mN} / \mathrm{m},{ }^{21}$ density $\rho=1144.7 \mathrm{~kg} / \mathrm{m}^{3}$, and refractive index $n=1.3677 .{ }^{14,22}$

variations were observed. Also shown in the plot are the resonant frequencies $f_{\text {res }}^{\text {th }}$ calculated from Eq. 1 for several contact angles with the contact-angle-dependent eigenvalues $\lambda_{1}(\theta)$ determined using previously described procedure. ${ }^{12}$ Comparison of the measured and calculated values of the lowest-mode resonant frequencies indicates that the droplet resonant behavior is indeed well described by the formalism developed by Strani and Sabeta in Ref. 11. The spread in the measured resonant frequencies for droplets of given $R$ can be attributed mainly to the spot-to-spot variations of the local chemistry and/or topography of the studied SH surface that are also observed in light microscopy, AFM, and SEM images of the surfaces ${ }^{23}$ and that lead to the contact angle hysteresis. ${ }^{24}$ Additional uncertainty of $f_{\text {res }}^{\text {exp }}$ then stems from the non-uniform response of the sample chamber to the mechanical oscillatory driving (see Figure 1).

From the experimental data presented in Figure 3, it is evident that the mechanical resonant frequencies for the droplets of a given $R$ differ for the LE1/ethanol-coated and LE1/chloroformcoated SH surfaces. Since the droplet material properties are identical for both cases, this deviation can only be attributed to contact angles differences between the two surfaces (see Eq. 1). Such dependence of the contact angle on the solvent used for the SH layer deposition has been previously 
(a)

(b)
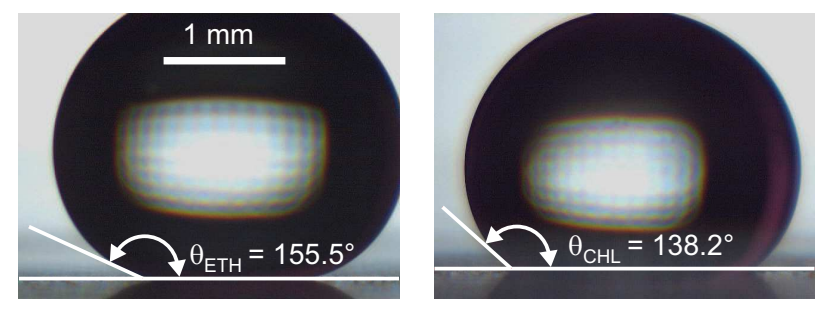

(c)

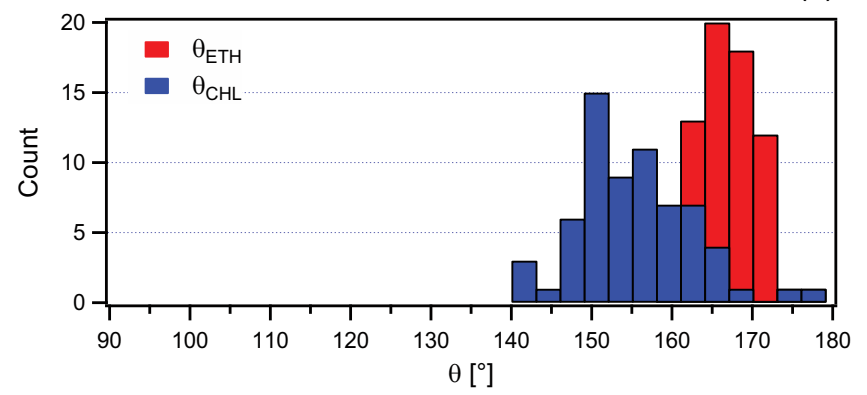

Figure 4: Contact angle measurements on superhydrophobic (SH) surfaces. Direct imaging of an $\mathrm{NaCl}$-water droplet on (a) LE1/ethanol-coated and (b) LE1/chloroform-coated SH surfaces. (c) Histogram of the microscopic contact angles $\theta$ for the LE1/ethanol-coated (70 measurements, $\left.\tilde{\theta}_{\mathrm{ETH}}=166.7^{\circ}\right)$ and LE1/chloroform-coated $\left(68\right.$ measurements, $\left.\tilde{\theta}_{\mathrm{CHL}}=153.8^{\circ}\right) \mathrm{SH}$ surfaces. The contact angles are calculated from the data presented in Figure 3 using Eq. 1.

reported. $^{25}$

Figure 4 summarizes the results of both macroscopic and microscopic (vibration-based) contact angle measurements on the two studied SH surfaces. Macroscopic values of $\theta$ were obtained using direct imaging of stationary droplets generated on a horizontal surface with a syringe (see examples in Figure $4 \mathrm{a}, \mathrm{b}$ ). This procedure yielded average contact angles $\bar{\theta}_{\mathrm{ETH}}=$ $(154.2 \pm 9.1)^{\circ}$ on the LE1/ethanol-coated SH surface $(4$ measurements $)$ and $\bar{\theta}_{\mathrm{CHL}}=(139.4 \pm 3.6)^{\circ}$ on the LE1/chloroform-coated SH surface (4 measurements), respectively. From the data of Figure 3, microscopic contact angles were calculated through Eq. 1. Their distributions are shown in Figure $4 \mathrm{c}$ with the median values of the contact angles being $\tilde{\theta}_{\mathrm{ETH}}=166.7^{\circ}$ on the LE1/ethanolcoated SH surface and $\tilde{\theta}_{\mathrm{CHL}}=153.8^{\circ}$ on the LE1/chloroform-coated SH surface, respectively. These values agree well with the ranges of contact angles determined for microdroplets on a similar SH surface using electrical deformation. ${ }^{26}$

As both studied SH surfaces are formed by layers of the same hydrophobic silica nanoparticles, 

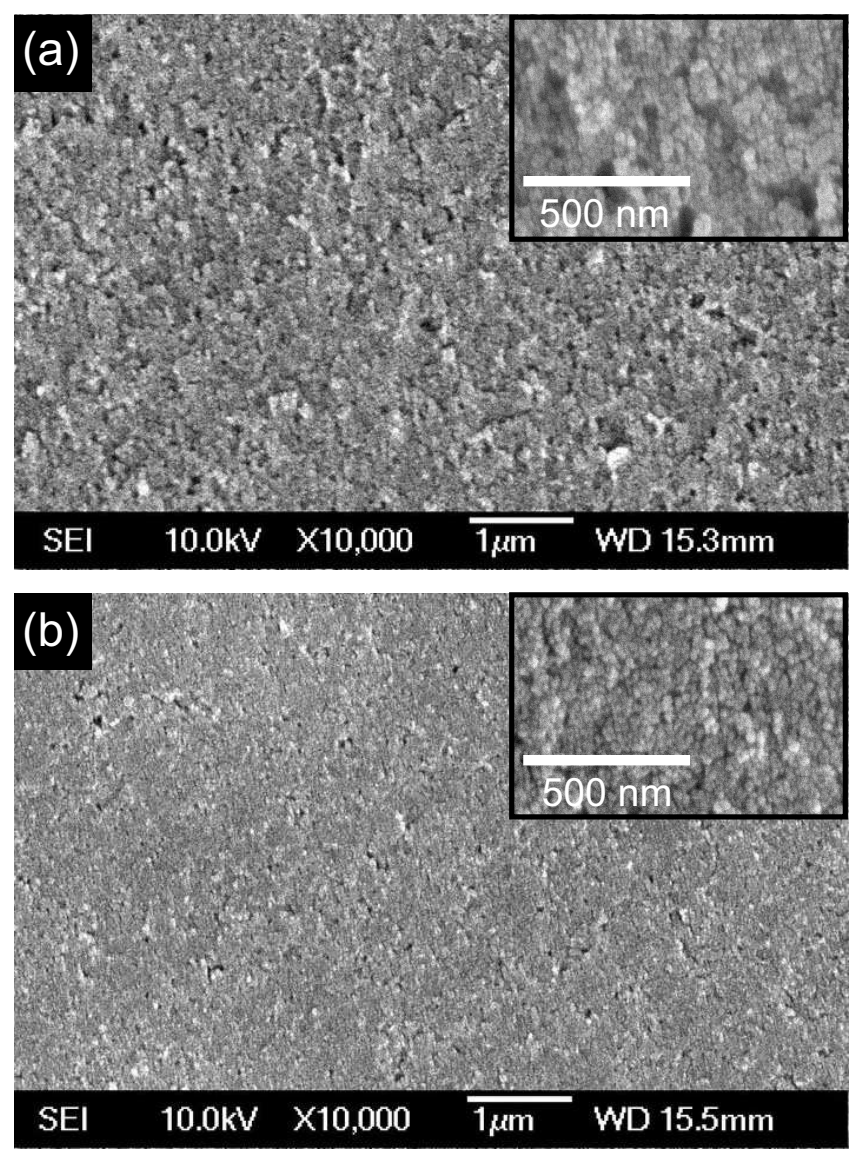

Figure 5: SEM images of the studied superhydrophobic surfaces. (a) LE1/ethanol-coated SH surface (b) LE1/chloroform-coated SH surface. Image insets show surface details obtained at higher magnification.

difference in their contact angles cannot be caused by their chemical properties. It is well established that SH surfaces with multiple scales of roughness display higher contact angles than more homogeneous surfaces with the same surface chemistry. ${ }^{27,28}$ Comparison of the SEM images of our model SH surfaces presented in Figure 5 reveals a significantly higher variations in roughness of the LE1/ethanol-coated surface relative to the LE1/chloroform-coated surface. Thus, the observed contact angle difference between the two surfaces indeed stems from the surface topography change.

Several groups have applied substrate vibrations in order to induce a wetting transition or contact angle relaxation in a sessile droplet deposited on a surface displaying contact angle hysteresis. ${ }^{29-31}$ In the measurements reported here, no such wetting transitions were observed, as verified 
by monitoring the WGM positions during a frequency sweep and also after the mechanical driving was turned off. If the droplet went through a wetting transition, its contact angle and, consequently, spherical radius would change abruptly resulting in an abrupt shift of the WGM positions. However, except for a few cases that were excluded from further analysis, no such abrupt changes were observed; small gradual drifts $(<0.5 \mathrm{~nm})$ of the mode spectral positions are due to temperature fluctuations. Thus, we can assume that the microscopic measurements reported here probe the (metastable) wetting states of the droplets arising at their generation time without changing them. As our SH surfaces display contact angle hysteresis, these initial droplet wetting states may not correspond to the global energy minimum (the lowest energy metastable state ${ }^{29}$ ).

Comparison of the macroscopic and microscopic contact angles reveals a difference in the two values with microscopic measurements being systematically shifted to larger values by about $14^{\circ}$. We attribute this to observing wetting states that are closer to the global energy minimum ${ }^{29}$ in macroscopic measurements where a droplet rolls easily on the surface and can readily move to the most hydrophilic region in the vicinity of the deposition point. Relaxation of the contact angle in these measurements is further facilitated by mechanical agitation during the syringe retraction.

From a practical point of view, it is worth noting that the microscopic contact angle values measured with small droplets are more appropriate for microfluidics applications as they reflect the wetting properties of the surfaces at the relevant length scale. For the typical droplet radius and contact angle values $\left(R=5 \mu \mathrm{m}, \theta=165^{\circ}\right)$ on a SH surface, the contact area between the droplet and the substrate has the radius $r=R \sin (\theta) \approx 1.3 \mu \mathrm{m}$; it is this radius that sets the spatial resolution limit of the contact angle measurement. We note that this resolution limit can be further reduced to $<1 \mu \mathrm{m}$ by employing smaller droplets for the surface probing. In order to accomplish this, a different PZT that allows efficient excitation of droplet vibrations above $200 \mathrm{kHz}$ has to be used.

In conclusion, we have characterized experimentally the microscopic liquid-solid contact angles by analyzing the mechanical resonances of individual micrometric liquid droplets standing on a vibrated SH surface. For two surfaces with different superhydrophobicity levels we have 
compared the contact angles determined with this method to the values obtained with macroscopic droplet imaging and found a good correlation between the microscopic and macroscopic contact angle measurements. Further investigations are needed to elucidate the relation between the measured microscopic contact angles and global wetting energy minimum for the studied substrateliquid combination. The presented microscopic contact angle probing has potential applications in the characterization of structured surfaces with complex surface chemistry patterning under a wide range of ambient atmospheric conditions, especially if combined with a precise on-demand positioning of the liquid droplets over the target surface areas.

\section{Acknowledgement}

This work is partially supported by TÜBİTAK (Grant No: 109T734), European Commission Marie Curie IEF (contract number PIEF-GA-2009-252579; A.J.), and FABED Young Investigator Research Award (A.K.). One of the authors (I.K.) further acknowledges support from the TÜBİTAK post-doctoral fellowship program.

\section{References}

(1) Nosonovsky, M.; Bhushan, B. Curr. Opin. Colloid. Interface Sci. 2009, 14, 270-280.

(2) Bhushan, B. Microelectronic Engineering 2007, 84, 387-412.

(3) Rothstein, J. P. Annu. Rev. Fluid Mech. 2010, 42, 89-109.

(4) Kiraz, A.; Kurt, A.; Dündar, M. A.; Demirel, A. L. Appl. Phys. Lett. 2006, 89, 081118.

(5) de Gennes, P. G. Rev. Mod. Phys. 1985, 57, 827-863.

(6) Lau, K. K. S.; Bico, J.; Teo, K. B. K.; Chhowalla, M.; Amaratunga, G. A. J.; Milne, W. I.; McKinley, G. H.; Gleason, K. K. Nano Lett. 2003, 3, 1701-1705. 
(7) Journet, C.; Moulinet, S.; Ybert, C.; Purcell, S. T.; Bocquet, L. Europhys. Lett. 2005, 71, 104-109.

(8) Hung, Y.-L.; Chang, Y.-Y.; Wang, M.-J.; Lin, S.-Y. Rev. Sci. Instrum. 2010, 81, 065105.

(9) Checco, A.; Guenoun, P.; Daillant, J. Phys. Rev. Lett. 2003, 91, 186101.

(10) Cheng, Y.-T.; Rodak, D. E.; Angelopoulos, A.; Gacek, T. Appl. Phys. Lett. 2005, 87, 194112.

(11) Strani, M.; Sabetta, F. J. Fluid Mech. 1984, 141, 233-247.

(12) Smithwick, R. W.; Boulet, J. A. M. J. Colloid Interface Sci. 1989, 130, 588-596.

(13) Yamakita, S.; Matsui, Y.; Shiokawa, S. Jpn. J. Appl. Phys. 1999, 38, 3127-3130.

(14) Kiraz, A.; Karadag, Y.; Yorulmaz, S. C.; Muradoglu, M. Phys. Chem. Chem. Phys. 2009, 11, $2597-2560$.

(15) Fields, M. H.; Popp, J.; Chang, R. K. Nonlinear Optics in Microspheres. In Progress in Optics; Wolf, E., Ed.; Elsevier, 2000; Vol. 41, pp 1-95.

(16) Noblin, X.; Buguin, A.; Brochard-Wyart, F. Eur. Phys. J. E 2004, 14, 395-404.

(17) Celestini, F.; Kofman, R. Phys. Rev. E 2006, 73, 041602.

(18) McHale, G.; Elliott, S. J.; Newton, M. I.; Herbertson, D. L.; Esmer, K. Langmuir 2009, 25, $529-533$.

(19) Eversole, J. D.; Lin, H.-B.; Campillo, A. J. Appl. Opt. 1992, 31, 1982-1991.

(20) Eversole, J. D.; Lin, H.-B.; Campillo, A. J. J. Opt. Soc. Am. B 1995, 12, 287.

(21) Marcus, Y. J. Chem. Eng. Data 2010, 55, 3641-3644.

(22) Lide, D. R. CRC Handbook of Chemistry and Physics, Internet Version 2005, $<$ http://www.hbcnetbase.com>; CRC Press, 2005. 
(23) Yüce, M. Y.; Demirel, A. L.; Menzel, F. Langmuir 2005, 21, 5073.

(24) Marmur, A. Advances in Colloid and Interface Science 1994, 50, 121-141.

(25) Yüce, M. Y. M.Sc. thesis, Koç University, 2006.

(26) Yorulmaz, S. C.; Mestre, M.; Muradoglu, M.; Alaca, B. E.; Kiraz, A. Opt. Commun. 2009, 282, 3024-3027.

(27) Nosonovsky, M. Langmuir 2007, 23, 3157-3161.

(28) Shirtcliffe, N. J.; McHale, G.; Newton, M. I.; Chabrol, G.; Perry, C. C. Adv. Mater. 2004, 16, 1929-1932.

(29) Mettu, S.; Chaudhury, M. K. Langmuir 2010, 26, 8131-8140.

(30) Meiron, T. S.; Marmur, A.; Saguy, I. S. J. Colloid Interface Sci. 2004, 274, 637-644.

(31) Bormashenko, E.; Pogreb, R.; Whyman, G.; Erlich, M. Langmuir 2007, 23, 12217-12221. 


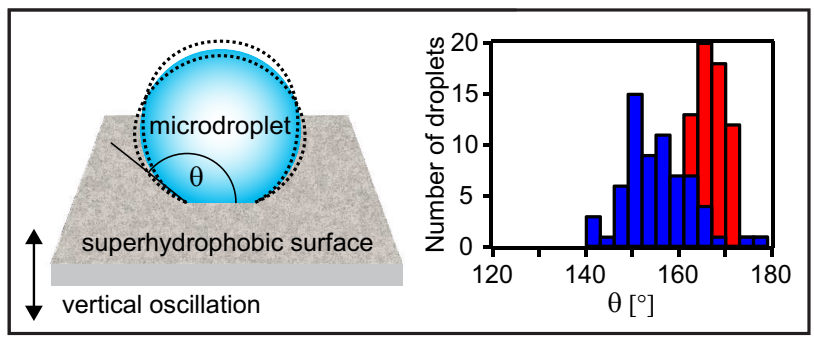

Figure 6: *

For table of contents only 